Andrew Young School of Policy Studies Research Paper Series

Working Paper 08-14

August 2008

Department of Economics

\title{
Coalition Decisions and Simple Majority Rule
}

\author{
Yongsheng $\mathrm{Xu}$ \\ Georgia State University
}

This paper can be downloaded at:

http://aysps.gsu.edu/publications/2008/index.htm

The Social Science Research Network Electronic Paper Collection:

http://ssrn.com/abstract=1238702 
Coalitional Decisions and Simple Majority Rule*

\author{
Yongsheng $\mathrm{Xu}$ \\ Department of Economics \\ Andrew Young School of Policy Studies \\ Georgia State University \\ Atlanta, GA 30303 \\ Email: yxu3@gsu.edu
}

This version: August 2008

* I thank Wulf Gaertner for helpful conversations. 


\begin{abstract}
We study simple majority rule from a perspective of coalitional decision makings. Four attrative properties each linking decisions by a group to decisions by its varioius coalitions are introduced, and are used for characterizing simple majority rule. Our characterization result provides an alternative to that of May (1952).

JEL Classification Numbers: D'7
\end{abstract}




\section{Introduction}

One of the best known voting procedures is simple majority rule: when a group of people deciding on two options and assuming that each individual casts one vote, the option that gets 'more' votes than the other emerges as the winner. Simple majority rule is fairly easy to understand and to implement, and has several attractive normative properties as studied in May (1952): it treats individuals symmetrically (the rule is anonymous), it is neutral with respect to options (there is no significance of names attached to the options), and it responds to individuals' preferences positively. ${ }^{1}$

In May's (1952) study of simple majority rule, he takes a group of individuals as fixed and allows their preferences to vary. In this paper, we take the preferences of a group of individuals as fixed and study simple majority rule from a perspective that links the group's decision with decisions made by its subgroups (coalitions). This is motivated by an observation that group decisions are essentially compromises among group members and/or among its various coalitions: a group's decision on two options depends on how its various coalitions decide on the two options. How exactly are coalitions' decisions linked to the group's decision? For example, when an option $x$ considered to be the winner for each of the two disjoint coalitions over another option $y$, would $x$ continue to be considered the winner for the group joined by the two coalitions? This is one of the possible links between a group of individuals and its coalitions that we intend to explore in this paper. In particular, we show that simple majority rule is characterized by the following properties (see formal definitions of these properties in Section 3): (a) if two disjoint coalitions each consider an option $x$ as the winner over another option $y$, then when they join into a single coalition, the option $x$ continues to be the winner over the option $y$ by the enlarged coalition; (b) whenever a coalition of a given group is indifferent between two options $x$ and $y$, the group's decision on $x$ and $y$ is determined by the other coalition after 'taking out' this indifference-coalition; (c) any coalition of two individuals with opposite views over two options $x$ and $y$ should express an indifference between the two options; (d) the decision by any coalition with just one individual must rest on this individual's preferences.

\footnotetext{
${ }^{1}$ Apart from May's (1952) paper, simple majority rule has been studied by many researchers from various perspectives. The most recent contribution is by Dasgupta and Maskin (2008) where the authors give an axiomatic characterization of simple majority rule over a larger domain of individual preferences than any other voting rules.
} 
In Section 2, we introduce our basic notation and definitions. Section 3 presents several attractive properties and gives an axiomatic characterizations for simple majority rule. The paper is concluded in Section 4 by making some brief remarks.

\section{Notation and Definitions}

Let there be $n \geq 2$ individuals and two alternatives $x$ and $y$. The set of individuals is to be denoted by $N$. For each $i \in N, R_{i}$ stands for individual $i$ 's preferences over $x$ and $y$. Let $P_{i}$ and $I_{i}$ stand, respectively, for the asymmetric and symmetric part of $R_{i}$.

Non-empty subsets of $N$ are denoted by $S, T, \cdots$, and are called coalitions. For any coalition $S, \# S$ denotes the cardinality of $S$.

Let $\alpha^{N} \equiv\left\{R_{1}, \cdots, R_{i}, \ldots, R_{n}\right\}$ denote a profile of individuals' preferences over $x$ and $y$. In this paper, we consider $\alpha^{N}$ as fixed. For any coalition $S$, let $\alpha^{S}$ denote the set $\left\{R_{i} \in \alpha^{N}: i \in S\right\}$.

An aggregation rule $f$ assigns, for each $\alpha^{S} \in \bigcup_{T \in \mathcal{N}} \alpha^{T}$, a complete binary relation $R\left(\alpha^{S}\right)$ over $x$ and $y: R\left(\alpha^{S}\right)=f\left(\alpha^{S}\right)$. The asymmetric and symmetric part of $R\left(\alpha^{S}\right)$ are denoted by $P\left(\alpha^{S}\right)$ and $I\left(\alpha^{S}\right)$, respectively.

\section{Simple Majority Rule}

For each coalition $S$, let $N\left(x, y ; \alpha^{S}\right) \equiv \#\left\{i \in S: x R_{i} y\right.$ for some $\left.R_{i} \in \alpha^{S}\right\}$. An aggregation rule $f$ is said to be simple majority rule iff, for all coalition $S, x f\left(\alpha^{S}\right) y \Leftrightarrow N\left(x, y ; \alpha^{S}\right) \geq N\left(y, x ; \alpha^{S}\right)$.

How is a group's decision linked with decisions made by its coalitions? We consider the following properties each linking a group's decision to its various coalitions.

Independence of an Unconcerned Coalition (IUC): For all coalitions $S$ and $T$ with $S \cap T=\emptyset$, if $x I\left(\alpha^{S}\right) y$, then $x R\left(\alpha^{T}\right) y \Leftrightarrow x R\left(\alpha^{S} \cup \alpha^{T}\right) y$.

Simple Equal Treatment (SET): For all $i, j \in N$, if $\left[x P_{i} y\right.$ and $\left.y P_{j} x\right]$ then $x I\left(\alpha^{\{i, j\}}\right) y$.

Monotonicity (M): For all coalitions $S$ and $T$ with $S \cap T=\emptyset$, if $\left[x P\left(\alpha^{S}\right) y\right.$ and $x P\left(\alpha^{T}\right) y$ ] then $x P\left(\alpha^{S} \cup \alpha^{T}\right) y$. 
Self Determination (SD): For all $i \in N, x R\left(\alpha^{\{i\}}\right) y \Leftrightarrow x R_{i} y$.

IUC requires that, for any two disjoint coalitions $S$ and $T$, whenever $S$ is indifferent between $x$ and $y$, the decision over $x$ and $y$ by the group, $S \cup T$, is determined by coalition $T$. SET says that, in a simple coalition consisting of two individuals, if they have opposite views over $x$ and $y$ (one prefers $x$ to $y$ and the other prefers $y$ to $x$ ), then this coalition should regard $x$ and $y$ as indifferent. SET reflects the idea that an aggregation rule should treat individuals equally in this simple situation. ${ }^{2} \mathrm{M}$ stipulates that, whenever two disjoint coalitions, $S$ and $T$, each regard $x$ as a better option than $y$, $x$ continues to be regarded better than $y$ by the coalition joined by $S$ and $T$. And finally, SD simply says that the decision by any coalition with just one member must be determined by its member. In a very weak sense, SD reflects an idea of self-determination in making decisions.

The following proposition presents our main result, a characterization of simple majority rule.

Proposition 1 An aggregation rule $f$ satisfies IUC, SET, $M$ and $S D$ if and only if it is simple majority rule.

Proof. It can be checked that simple majority rule satisfies IUC, SET, M and SD. We now show that, if an aggregation rule $f$ satisfies IUC, SET, M and $\mathrm{SD}$, then it must be simple majority rule.

Let $f$ be an aggregation rule satisfying IUC, SET, M and SD. We first note that, by $\mathrm{SD}$, it follows that

$$
\text { for all coalition } S=\{i\}, x R_{i} y \Leftrightarrow x R\left(\alpha^{S}\right) y
$$

Consider any coalition $S=\{i, j\}$ where $i$ and $j$ are distinct. We note that, for $a, b \in\{x, y\}$, if $a P_{i} b$ and $b P_{j} a$, then, by SET, $a I\left(\alpha^{S}\right) b$ follows immediately; if $a P_{i} b$ and $a P_{j} b$, then, from (1), we must have $a P\left(\alpha^{\{i\}}\right) b$ and $a P\left(\alpha^{\{j\}}\right) b$; a simple application of $\mathrm{M}$ gives us $a P\left(\alpha^{S}\right) b$; if $a P_{i} b$ and $a I_{j} b$, then, from (1), we have $a P\left(\alpha^{\{i\}}\right) b$ and $a I\left(\alpha^{\{j\}}\right) b$; by IUC, $a P\left(\alpha^{S}\right) b$ follows easily from IUC. Therefore, we obtain

\footnotetext{
${ }^{2}$ SET resembles an idea behind a simplification procedure discussed by Gaertner (1988) for reducing originally given profiles of preferences to their equivalent profiles of preferences.
} 
for all $S=\{i, j\}$ and all $a, b \in\{x, y\}$, if $\left[\left(a P_{i} b \& a I_{j} b\right)\right.$ or $\left.\left(a P_{i} b \& a P_{j} b\right)\right]$ then $a P\left(\alpha^{S}\right) b$, and if $\left[\left(a I_{i} b \& a I_{j} b\right)\right.$ or $\left.\left(a P_{i} b \& b P_{j} a\right)\right]$ then $a I\left(\alpha^{S}\right) b$.

Suppose that, for all coalition $S$ with $\# S \leq 2, f\left(\alpha^{S}\right)$ is given by simple majority rule. We show that for all coalition $T$ with $\# T=\# S+1, f\left(\alpha^{T}\right)$ is given by simple majority rule as well. Let $T$ be a coalition such that $\# T=\# S+1$. We distinguish four cases: (i) for some $i \in T, x I_{i} y$; (ii) for some $j, k \in T, x P_{j} y$ and $y P_{k} x$; (iii) for all $i \in T, x P_{i} y$; and (iv) for all $i \in T$, $y P_{i} x$.

Consider case (i) first. Note that in this case, for some $i \in T, x I_{i} y$. From (1), $x I\left(\alpha^{\{i\}}\right) y$. By IUC, we then have $x R\left(\alpha^{T}\right) y \Leftrightarrow x R\left(\alpha^{T-\{i\}}\right) y$. From our induction hypothesis, $x R\left(\alpha^{T-\{i\}}\right) y \Leftrightarrow N\left(x, y ; \alpha^{T-\{i\}}\right) \geq N\left(y, x ; \alpha^{T-\{i\}}\right)$. Therefore, from $x R\left(\alpha^{T}\right) y \Leftrightarrow x R\left(\alpha^{T-\{i\}}\right) y$ and $x I_{i} y$, it follows that $x R\left(\alpha^{T}\right) y \Leftrightarrow N\left(x, y ; \alpha^{T}\right) \geq N\left(y, x ; \alpha^{T}\right)$.

Consider case (ii) in which for some $j, k \in T, x P_{j} y$ and $y P_{k} x$ next. If $x P_{j} y$ and $y P_{k} x$ for some $j, k \in T$, then, from (2), $x I\left(\alpha^{\{j, k\}}\right) y$. By IUC, we then have $x R\left(\alpha^{T}\right) y \Leftrightarrow x R\left(\alpha^{T-\{j, k\}}\right) y$. From our induction hypothesis, $x R\left(\alpha^{T-\{j, k\}}\right) y \Leftrightarrow N\left(x, y ; \alpha^{T-\{j, k\}}\right) \geq N\left(y, x ; \alpha^{T-\{j, k\}}\right)$. Then, $x R\left(\alpha^{T}\right) y \Leftrightarrow$ $N\left(x, y ; \alpha^{T}\right) \geq N\left(y, x ; \alpha^{T}\right)$ follows from $x R\left(\alpha^{T}\right) y \Leftrightarrow x R\left(\alpha^{T-\{j, k\}}\right) y$ and $\left[x P_{j} y\right.$ and $\left.y P_{k} x\right]$

Thirdly, we consider case (iii) where $x P_{i} y$ for all $i \in T$. Note that, in this case, $N\left(x, y ; \alpha^{T}\right)=\# T$ and $N\left(y, x ; \alpha^{T}\right)=0$. From our induction hypothesis, we must have $x P\left(\alpha^{T-\{j\}}\right) y$ and $x P\left(\alpha^{\{j\}}\right) y$ for some $j \in T$. Therefore, by M, it follows that $x P\left(\alpha^{T}\right) y$.

Case (iv) in which for all $i \in T, y P_{i} x$, is similar to case (iii), and it can be shown that $y P\left(\alpha^{T}\right) x$.

The above cases exhaust all possibilities. Therefore, combining (1) and (2), we have shown that

for all coalition $S, x R\left(\alpha^{S}\right) y \Leftrightarrow N\left(x, y ; \alpha^{S}\right) \geq N\left(y, x ; \alpha^{S}\right)$.

This completes the proof of Proposition 1.

It may be noted that, in the above result, Monotonicity can be replaced by the following Unanymity property:

Unanymity (U): For all coalitions $S$, if $\left[x P_{i} y\right.$ for all $\left.i \in S\right]$ then $x P\left(\alpha^{S}\right) y$. 
$\mathrm{U}$ says that if every individual in a coalition prefers $x$ to $y$, then the coalition must rank $x$ better than $y$. This is the weak Pareto principle in our context.

\section{Conclusion}

In this paper, we have studied simple majority rule from the perspective of coalitional decision makings. Our main intention is to explore the link between decision makings by a group and its various coalitions. In this context, we have provided an alternative characterization for simple majority rule.

To the best of our knowledge, our study of simple majority rule is the first to be conducted in the framework proposed in this paper. It would be interesting to study other well-known voting rules in this or similar frameworks.

\section{References}

Dasgupta, P. and E. Maskin (2008). "On the robustness of majority rule," Journal of European Economic Association, 6(5), 949-973.

Gaertner, W. (1988). "Binary inversions and transitive majorities", in Measurement in Economics, W. Eichhorn (ed.), Physica-Verlag, Heidelberg, 253-267.

May, K.O. (1952). "A set of independent necessary and sufficient conditions for simple majority decisions," Econometrica, 20(4), 680-684. 